$\Delta p$, namely [see Eq. (6)]

$$
\Delta p^{0}=7.163 m_{0} c \text {. }
$$

Similarly, the proper value $\Delta q^{0}$ of the diameter for an inside observer is [see Eq. (8)]

$$
\Delta q^{0}=0.881 D \text {. }
$$

Whereas (1) $\left(1^{\prime}\right)$ give the product

$$
\Delta q \Delta p=2 \pi D m_{0} c\left(\frac{3}{4} 137\right),
$$

(2) $\left(2^{\prime}\right)$ give the product of order

$$
\Delta q^{0} \Delta p^{0}=2 \pi D m_{0} c
$$

in which the factor $\left(\frac{3}{4} 137\right)$ has disappeared. Thus, an electric particle can be characterized as a distribution in space and momentum space such that the product of the widths (uncertainty product) $\Delta q \Delta p=h$ reduces to a proper uncertainty product $\Delta q^{0} \Delta p^{0}=h_{0}$ which is of the order. of 137 times as small. The arbitrariness in assuming a certain value for the "diameter" of the particle has been removed by a more consistent calculation to be published later.

We are now going to report the calculations that have led to the proper values $\Delta p^{0}$ and $\Delta q$ quoted in $(2)\left(2^{\prime}\right)$. In the dimensionless quantities

$$
P=p / m_{0} c \text { and } E=\epsilon / m_{0} c^{2},
$$

the Einstein energy-momentum equation reads

$$
E^{2}=1+P^{2} \quad \text { with } \quad E d E=P d P .
$$

A line element $d P$ is the projection of a line element $\left(d P^{2}+d E^{2}\right)^{\frac{1}{2}}$ on the hyperbola (5). Its proper length is obtained by dividing it by the proper unit of length which is its distance $\left(P^{2}+E^{2}\right)^{\frac{1}{2}}$ from the zero point. Integrating we obtain the proper increase

$$
\begin{aligned}
\Delta P^{0}=\int\left(\frac{d P^{2}+d E^{2}}{P^{2}+E^{2}}\right)^{\frac{1}{2}} & =\int_{0}^{\Delta P} \frac{d P}{\left(1+P^{2}\right)^{\frac{1}{2}}} \\
= & \ln \left\{\Delta P+\left(1+\Delta P^{2}\right)^{\frac{1}{2}}\right\}=\operatorname{Sinh}^{-1} \Delta P .
\end{aligned}
$$

Substituting $\Delta P$ from (1) we obtain the result (2). Born's idea of reciprocity has led us to consider a similar reduction in space. Starting from the equation

$$
(c t)^{2}=D^{2}+q^{2}, \text { hence } d q / d t=c \cdot\left[1+(D / q)^{2}\right]^{\frac{1}{2}},
$$

and introducing the dimensionless coordinates

$$
Q=q / D \text { and } T=c t / D
$$

we obtain in the same way as in (6)

$$
T^{2}=1+Q^{2} \text { and } \Delta Q^{0}=\operatorname{Sinh}^{-1} \Delta Q .
$$

Then, using $\Delta q=D$ or $\Delta Q=1$ we obtain the result of $\left(2^{\prime}\right)$. The Eq. (7) is the mathematical formulation of an important result of Dirac's that a signal traveling from infinity to the center of the electron saves time as against a signal traveling with velocity $c$.

$$
\begin{gathered}
\text { Mendenhall Laboratory, } \\
\text { Ohio State University, } \\
\text { Columbus, Ohio, } \\
\text { June 7, 1939. }
\end{gathered}
$$

\section{On the Collimation of Fast Neutrons}

Several experiments ${ }^{1,2}$ have been carried out with a beam of neutrons produced by placing a thick wall of water in front of a neutron source and using a hole through the wall as a collimator. It has been observed with cloud chambers $^{1}$ that while there are large numbers of hydrogen recoils produced with the chamber in the beam, the number and length are both greatly diminished if the chamber is moved out of the beam. It has also been observed ${ }^{2}$ that such a collimating system supplemented by lead shields to cut down $\gamma$-radiation from the collimating screen, gives little ionization current when the chamber is taken out past the edge of the beam, compared to that produced in the beam.

The effects of neutron collimation are rather clearly indicated in the accompanying photographs which show the proton recoils produced in a cloud chamber filled to atmospheric pressure with $\mathrm{CH}_{4}$. The neutrons were produced by the bombardment of lithium or beryllium with $1.2-\mathrm{Mev}$ deuterons accelerated by a cyclotron. A wall of water which was about $75 \mathrm{~cm}$ thick in the region of the hole served as the collimator. This hole was about $10 \mathrm{~cm}$ in

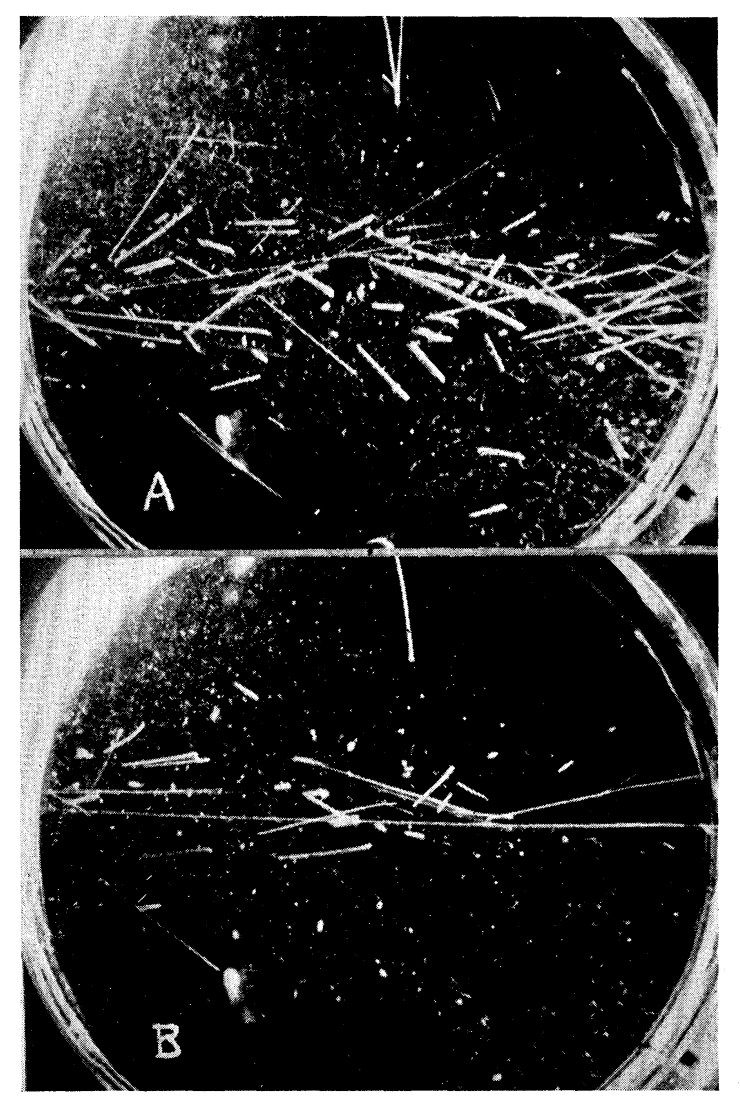

FIG. 1A. Tracks of the recoils in $\mathrm{CH}_{4}$ produced by a collimated beam of neutrons which passed from left to right through the cloud chamber. Left edge of chamber about $4 \mathrm{~cm}$ from collimator. B. Tracks of recoils produced when the nearest edge of chamber was $30 \mathrm{~cm}$ from the collimator. 
diameter but was stopped down by the insertion of paraffin cylinders with central holes of smaller diameter. These pictures were taken with such a collimator which had a diameter of about $4 \mathrm{~cm}$. In order to avoid unnecessary neutron scattering by the chamber or camera support, they were of light-weight construction throughout. The cloud chamber (diameter $22 \mathrm{~cm}$ ) was of the rubber diaphragm type with the regulator valve located about $40 \mathrm{~cm}$ below the chamber. The light source was a projection lamp with collimating lenses and also located at a distance from the cloud chamber.

Figure $1 \mathrm{~A}$ shows the recoils produced by the neutrons from a lithium target. The neutron beam entered the chamber on the left side which was about $4 \mathrm{~cm}$ from the end of the collimator. Most of the recoils appear to originate in a region which has about the same width as the collimator. At the top of the photograph there appears a wire projecting from the wall of the chamber, on the end of which a weak polonium source had been placed. Fig. 1A shows part of an $\alpha$-track from this source. Fig. 1B shows the recoils produced by the neutrons from a beryllium target when the nearest edge of the chamber was about 30 $\mathrm{cm}$ from the end of the collimating tube indicating that the beam shows no obvious divergence.

R. F. BACHER

D. C. SWANSON*

Cornell University,

August 4, 1939.

* At present at the University of Florida.

1 R. F. Bacher and D. C. Swanson, Phys. Rev. 53, 676 (1938); 53 922 (1938).

${ }^{2}$ P. C. Aebersold, Phys. Rev. 55, 596 (1939); P. C. Aebersold and Gladys A. Anslow, Phys. Rev. 55, 680 (1939); 55, 1134 (1939).

\section{A Nuclear Disintegration Induced by the Cosmic Radiation}

The presence of heavy ionizing particles in the cosmic radiation has been observed on several occasions and ascribed to nuclear disintegrations taking place under the action of primary cosmic rays. Since the first observation by Anderson ${ }^{1}$ different authors have observed cloud tracks attributable to phenomena of this kind. ${ }^{2}$ The occurrence of such tracks, very rare at sea level, is much more frequent at high altitudes; the tracks originate either in the metallic parts of the Wilson chamber or in its gaseous atmosphere. The identification of the nature of these particles was generally very uncertain because of the difficulties, often insuperable, of determining the actual direction of their movement and of measuring with any exactitude their range and the curvature of their tracks in a magnetic field.

In most cases however, the properties of protons are in better agreement than those of any other kind of particles with the bulk of recorded observations. A similar conclusion, that is, the occurrence of disintegrations induced by the cosmic rays and resulting in an emission of protons, seems to be suggested by recent experiments with photographic emulsion. ${ }^{3}$

The accompanying photograph (Fig. 1) was taken in a horizontal cloud chamber subjected to a field of 16,400 oersteds. The object of our study was the $\alpha$-radiation from

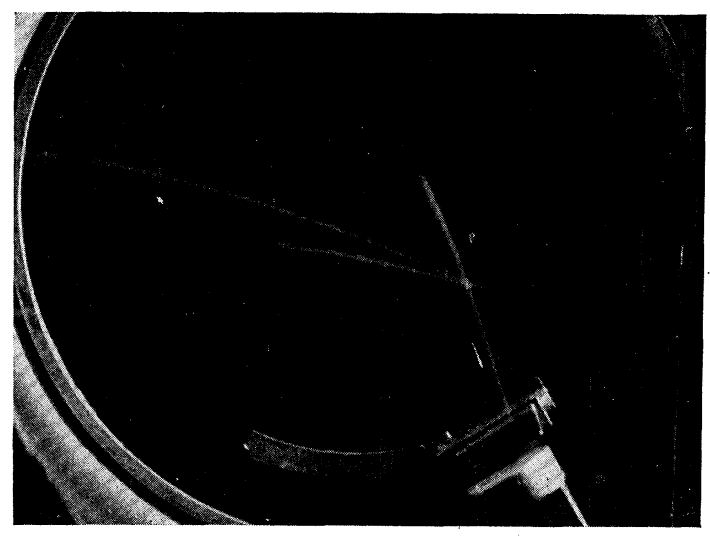

IIG. 1. Cloud-chamber photograph showing a polonium $\alpha$-particle track together with two tracks produced by cosmic rays in the cloudchamber atmosphere. The long track is due indubitably to a proton of $6.3 \mathrm{Mev}$. The short one is due either to a proton of $3.7 \mathrm{Mev}$ or a deuteron
of $2.0 \mathrm{Mev}$ energy.

Po, canalized into a narrow beam (aperture $10^{\circ}-15^{\circ}$ ). The photograph shows one of these $\alpha$-tracks accompanied by two tracks of another kind which must be ascribed to particles originated in a nuclear reaction induced by the cosmic rays in the chamber atmosphere.* Since the actual direction of these particles is from right to left, they must be positive. We determined the track curvature after the method (described elsewhere) ${ }^{4}$ of mean radii of curvature using an enlargement of the negative taken with a single objective. The lighted belt in the chamber being $2.5 \mathrm{~cm}$ high, the minimum inclination of the tracks relative to the field direction is $75^{\circ}$ for the long track and $55^{\circ}$ for the short one. We have determined several $H \rho$ values for different points of the two tracks (see Table I).

The ionization visible on these two tracks is 3 to 4 times less intense than that produced by the Po alphas. The tracks are due, therefore, neither to alphas nor to electrons. Even mesons are excluded, since a meson of a mass $M=200 m_{0}$ and of a $H \rho$ falling between $2.50 \times 10^{5}$ and $3.50 \times 10^{5}$ ought to produce an ionization 10 to 15 times less intense than the ionization observed. The tracks could be, however, ascribed to protons or deuterons. A detailed analysis of the curvature shows indeed that the long track is due indubitably to a proton and that the short one is due either to a proton or to a deuteron; the precision of measurements in this latter case being, however, insufficient to ensure an absolutely univocal identification.

The initial energy of the proton (long track) is equal to $6.3 \pm 0.2 \mathrm{Mev}$. The second particle (short track) possesses an initial energy of $3.7 \pm 0.8 \mathrm{Mev}$ if a proton, and of $2.0 \pm 0.3 \mathrm{Mev}$ if a deuteron. The total energy of the particles projected in the nuclear reaction we are discussing, must be, therefore, of the magnitude order of $10 \mathrm{Mev}$.

Assuming that the two tracks are due to two protons or to one proton and one deuteron, we may draw some conclusions as to the energy of the incident rays primarily responsible for the observed nuclear process. Anyone of the reactions which could take place in a cloud-chamber atmosphere consisting of air, water vapor and alcohol 


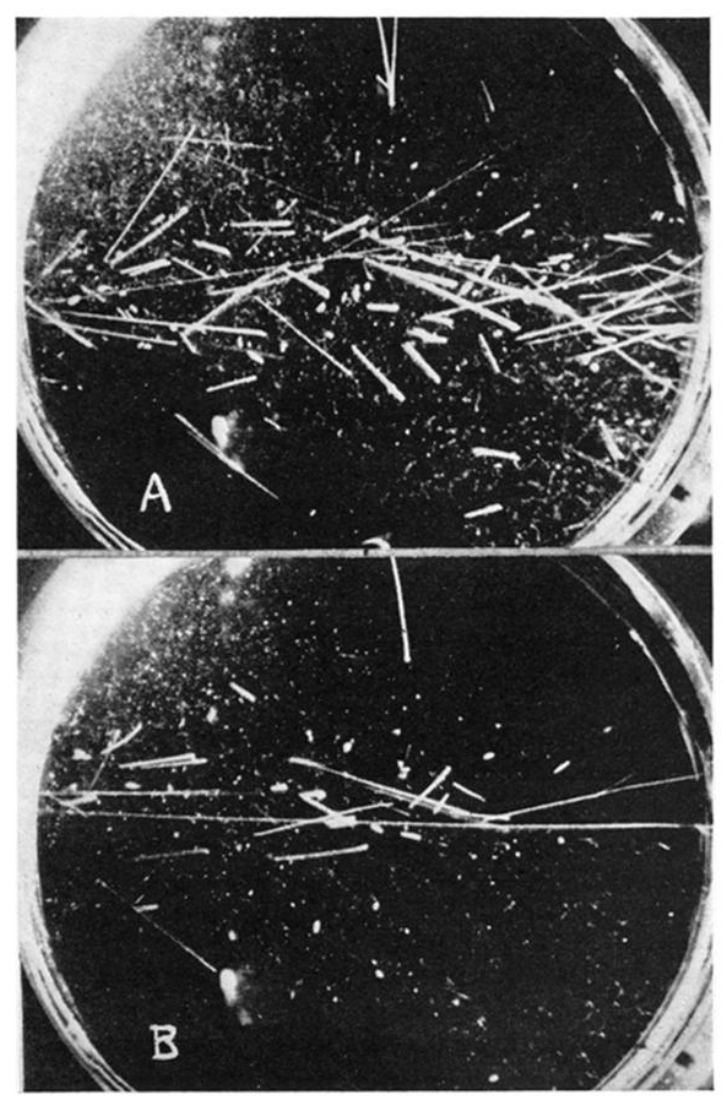

Fig. 1A. Tracks of the recoils in $\mathrm{CH}_{4}$ produced by a collimated beam of neutrons which passed from left to right through the cloud chamber. Left edge of chamber about $4 \mathrm{~cm}$ from collimator. B. Tracks of recoils prollumator. Whe the nearest edge of chamber was $30 \mathrm{~cm}$ from the collimator. 